\title{
La necesidad de la neuroética en la investigación de mercados. Estudio del Código de Ética de la Neuromarketing Science and Business Association
}

\author{
VIOLETA CORONA-CABRERA ${ }^{a}$
}

RESUMEN La contribución que las neurociencias han hecho para construir un conocimiento profundo en aspectos fundamentales del ser humano es incuestionable. Al contar con un mayor entendimiento del nivel neurofisiológico del hombre, hemos podido también deducir el origen de las percepciones, las emociones y, consecuentemente, de la conducta. Entender las motivaciones propias de determinado comportamiento ha representado para la investigación de mercados obtener insights implícitos, lo cual es un enorme avance en comparación con las metodologías tradicionales que únicamente permiten tener una visión explícita de los sujetos de estudio. Si bien los aportes de las neurociencias son abundantes, es necesario regular qué se puede hacer en relación con qué se debe hacer. Este artículo trata de la ética de la neurociencia en procesos comerciales, entendida como un marco ético que permite regular la investigación neurocientífica vinculada al marketing. Se parte de conceptos epistemológicos y antropológicos básicos para, posteriormente, examinar el código de ética de la Neuromarketing Science and Business Association, organismo que agrupa al mayor número de profesionales de investigación neurocientífica aplicada al marketing. Con esto se pretende poner de manifiesto la necesidad de complementar este marco regulatorio con principios de ética universales.

PALABRAS CLAVE ética, investigación, neuromarketing, neurociencias, neuroética.

\section{HISTORIA DEL ARTÍCULO}

¿CÓMO CITAR?:

Corona-Cabrera, V. (2017). La necesidad de la neuroética en la investigación de mercados. Estudio del Código de Ética de la Neuromarketing Science and Business Association. Perspectiva Empresarial, 5(1), 43-52. http:// dx.doi.org/10.16967/rpe.v5n1a4

RECIBIDO: 27 de noviembre de 2017 APROBADO: 15 de febrero de 2018

CORRESPONDENCIA:

Violeta Corona, Calzada

Circunvalación Poniente No. 49 Ciudad Granja CP 45010 Zapopan, Jalisco, México.

a Magíster, Profesora de la Universidad Panamericana, México. Correo electrónico: vcorona@up.edu.mx 


\section{¿CóMO CITO EL ARTÍ́CULO? HOW TO CITE THIS PAPER?}

CHICAGO:

Corona-Cabrera, Violeta. 2018.

"La necesidad de la neuroética en la investigación de mercados. Estudio del Código de Ética de la Neuromarketing Science and Business Association". Perspectiva Empresarial 5(1): 43-52. http:// dx.doi.org/10.16967/rpe. v5n1a4

MLA:

Corona-Cabrera, Violeta. "La necesidad de la neuroética en la investigación de mercados. Estudio del Código de Ética de la Neuromarketing Science and Business Association". Perspectiva Empresarial 5.1 (2018): 43-52. Digital. http:// dx.doi.org/10.16967/rpe. v5n1a4

\section{The Need for Neuroethics in Market Research. Study of the Neuromarketing Science and Business Association's Code of Ethics}

ABSTRACT The contribution that neurosciences have made to build deep knowledge of fundamental aspects of the human being is unquestionable. In having a greater understanding of the human neurophysiological level, we have also been able to deduce the origin of perceptions, emotions and, consequently, behavior. Understanding the motivations of certain behavior has represented implicit insights for market research, which is a huge advance compared to traditional methodologies that only allow an explicit vision of the subjects of study. Although the contributions of neurosciences are plentiful, it is necessary to regulate what can be done in relation to what should be done. This article deals with the ethics of neuroscience in business processes, understood as an ethical framework that regulates marketing-related neuroscientific research. It starts from basic epistemological and anthropological concepts to subsequently examine the code of ethics of the Neuromarketing Science and Business Association, an organization that brings together the largest number of professionals in neuroscientific research applied to marketing. This aims to highlight the need to complement this regulatory framework with universal ethical principles.

KEYWORDS ethics, research, neuromarketing, neurosciences, neuroethics.

\section{A necessidade da neuroética na pesquisa de mercados. Estudo do Código de Ética da Neuromarketing Science and Business Association}

RESUMO A contribuição que as neurociências ofereceram para construir um conhecimento profundo em aspectos fundamentais do ser humano é inquestionável. Ao contar com um maior entendimento do nível neurofisiológico do homem, pudemos também deduzir o origem das percepções, as emoções e, consequentemente, da conduta. Entender as motivações próprias de determinado comportamento representou, para a pesquisa de mercados, obter insights implícitos, o qual é um enorme avance em comparação com as metodologias tradicionais que unicamente permitem ter uma visão explícita dos sujeitos de estudo. Se por um lado os aportes das neurociências são abundantes por outro é necessário regular o que pode ser feito em relação com o que deve ser feito. Este artigo trata da ética da neurociência em processos comerciais, que é entendida como um marco ético que permite regular a pesquisa neurocientífica vinculada ao marketing. Parte-se dos conceitos epistemológicos e antropológicos básicos para, posteriormente, examinar o código de ética da Neuromarketing Science and Business Association, organismo que agrupa o maior número de profissionais de pesquisa neurocientífica aplicada ao marketing. Com isso, pretende-se manifestar a necessidade de complementar este marco regulatório com princípios de ética universais.

PALAVRAS CHAVE ética, pesquisa, neuromarketing, neurociências, neuroética. 


\section{Introducción}

A partir del siglo XXI las ciencias administrativas volcaron su interés hacia el estudio de las emociones y cómo estas influyen en la toma de decisiones (Beckman, 2008). De ahí surge la economía comportamental, la cual constituye la base del entendimiento de la conducta de consumo. Si bien el objetivo siempre ha sido el mismo (conocer los motivadores e influenciadores de decisión), los términos bajo los cuales se estudia al ser humano en pro de alcanzar esa comprensión han evolucionado a lo largo de los años. Es así como se pasó del enfoque de actitudes hacia la satisfacción y, posteriormente, se buscó con un afán extraordinario el cumplimiento de las expectativas. Indagar en los disparadores afectivos del consumidor es hoy en día tan importante como detectar el origen de sus pensamientos (Filser, 2000). Gerald Zaltman, profesor de la Universidad de Harvard, asegura que el $95 \%$ de todo el pensamiento que impulsa nuestro comportamiento ocurre en nuestra inconsciencia (Zaltman, 2003).

Los métodos tradicionales de investigación de mercados únicamente nos permiten acceder al nivel consciente de la toma de decisiones, por lo que ha sido necesario desarrollar nuevas metodologías de diagnóstico que exigen establecer una fuerte alianza entre las neurociencias y la investigación de mercados. Los procedimientos neurocientíficos han facilitado el entendimiento de los fundamentos del comportamiento del hombre mediante el estudio del sistema nervioso (Plassmann, Ramsøy \& Milosavljevic, 2012), y ha sido justo a raíz de su aplicación a la mercadotecnia que ha sido posible diseñar soluciones realmente eficaces.

Pareciera que estamos ante una nueva era de optimización del consumo, pues si sabemos lo que el consumidor piensa y quiere, entonces difícilmente nos equivocaremos al momento de satisfacer sus expectativas. Pero es entonces cuando surge la siguiente pregunta: ¿Qué tan lícito resulta llegar a ese nivel de conocimiento de los sujetos de estudio si se sabe de antemano que podremos revelar cuestiones que hasta este momento han permanecido veladas - por lo menos a nivel consciente- para la persona? En un afán por establecer límites y parámetros de acción en la práctica de las neurociencias, han surgido diferentes códigos de ética, sin embargo, al ser la mercadotecnia una vertiente relativamente joven en el área de las neurociencias, se considera que el rigor de su estructura y aplicación no cumple con las exigencias que requiere el estudio del hombre.

\section{Conceptos epistemológicos y antropológicos}

La neurociencia ostenta desde sus inicios la categoría de una nueva ciencia, cuyo alcance la faculta para hacer contribuciones sustanciales a la reflexión en torno a la neurofilosofía o neuroética (Comins-Mingol \& París-Albert, 2013). Al tratar particularmente de la neuroética, podemos encontrar dos líneas de estudio: la ética de la neurociencia y la neurociencia de la ética (Roskies, 2002).

La ética de la neurociencia experimenta con la dimensión fundamentalmente práctica de los discernimientos éticos relativos a la aplicación de la neurociencia, tanto en la relación médico-paciente como en su utilización con los sujetos experimentales, y en las implicaciones que pueden tener en la sociedad las nuevas técnicas de neuromarketing.

La ética, entendida en este sentido, se encarga de estudiar los actos de las neurociencias no como son, sino como deberían ser, y considera aspectos profundos, objetivos y universales característicos de la naturaleza específicamente humana.

Si nos referimos, en cambio, a la neurociencia de la ética, hablaríamos entonces del impacto del conocimiento neurocientífico en nuestra comprensión de la ética misma, es decir, cuáles son las bases neuronales de la agencia moral. Si como afirman Rosenzweig \& Leiman (2005), todos los pensamientos, sentimientos, percepciones y actos son resultado del sistema nervioso, habría que comprender entonces cómo se configura ese sistema nervioso para, a partir de ello, calificar moralmente los actos humanos. Este punto resulta fundamental al reflexionar que si no hay conciencia, no hay responsabilidad moral en el acto, por lo cual tendríamos que saber cuáles de nuestros actos son conscientes y cuáles no lo son. A fin de clarificar este interrogante, podemos hacer referencia a Denton, quien explica cómo sabemos que somos conscientes cuando podemos distinguir entre qué pensamientos se encuentran en nuestra cabeza y qué información sensorial acaece en el mundo exterior (Denton, 2009).

Para identificar si la práctica de las neurociencias es ética, podemos tomar como punto de 
partida los elementos en los cuales podemos detectar si un acto es bueno desde el punto de vista moral, los cuales son: objeto, fin y circunstancias. En cuanto a objeto, habría que establecer cuál es la naturaleza de la obra considerada en sí misma. En relación con el fin, determinar la intención con que se obra, y en cuanto a las circunstancias, indagar en todos los elementos accidentales que rodean la acción. En el propósito de que las neurociencias se practiquen con ética, los tres elementos deben ser buenos.

El objeto de estudio de las neurociencias es el sistema nervioso, lo cual implica profundizar en las sensaciones, las percepciones, las emociones, el lenguaje, la memoria, la conciencia, la toma de decisiones y las representaciones (Sanguineti, 2015). En el cerebro prevalece la morada de las funciones cognitivas y morales del individuo. Es necesario contar con el registro biométrico de las emociones, pero del mismo modo es preciso realizar la inclusión de un entendimiento profundo que demuestre cómo la persona percibe una situación. Solo con esta doble interpretación podrá concebirse una aproximación a la comprensión real de las emociones (Ramos-Zúñiga, 2014). Como se ha demostrado, el objeto de estudio de las neurociencias es muy amplio puesto que abarca de cierta forma todo lo humano. Así, entonces, dicho objeto es bueno, ya que el cerebro es importante como parte de una estructura unitaria de la persona. En una visión tomista, alma y cuerpo son constitutivos esenciales de la persona humana, ya que esta no es el alma sola, ni el cuerpo solo; y el alma no puede realizar su esencia sin el cuerpo (Santo Tomás). En consecuencia, no existe una acción humana que no se relacione con alguna actividad cerebral, por lo que el carácter de las neurociencias es psíquico y biológico.

Una vez se demuestra que el objeto de las neurociencias es bueno, habría que detenernos a reflexionar en su fin, el cual puede ser variable dependiendo de la intencionalidad de quienes la apliquen. Cuando el fin que persiguen las neurociencias al descubrir las correlaciones entre lo psíquico y lo humano se relaciona con el hedonismo, es decir, con la búsqueda del placer instantáneo, o con el consumismo, que es sustituir unos bienes por otros supuestamente mejores, entonces no podríamos hablar de una práctica ética (Llano \& Zagal, 2001). En el primer caso, contribuiríamos a formar a un hombre egoísta, sin dominio de sí mismo; en el segundo, construiríamos una falsa ilusión de distinción cimentada en la moda, en algo pasajero y transitorio que jamás podrá ser compatible con los valores trascendentes.

Al remitirnos al estudio de las neurociencias desde una perspectiva comercial, es aconsejable dilucidar el fin de la mercadotecnia. Cuando hablamos de mercadotecnia, resulta difícil no caer en la eterna discusión de su fin. Muchos afirman que la mercadotecnia crea necesidades, sin embargo, esto no es posible si se entiende que las necesidades ya existen y lo único que hacen las estrategias comerciales es proponer nuevas maneras de satisfacerlas, o bien generar estímulos que despierten deseos. Esta confusión quizá nace de la falta de claridad entre querer y desear. Los deseos se encuentran a un nivel emocional y son sugerencias de algo que podríamos llegar a querer, pero no son quereres efectivos. Al discutir sobre el querer, hacemos alusión a una inclinación de nuestra voluntad, y en esa tendencia evolutiva no puede interferir la mercadotecnia, ni tampoco las neurociencias. Decir lo contrario sería caer en un determinismo, o lo que es lo mismo, negar que la persona es un agente capaz de acción, dirigido hacia el futuro. Por tanto, si el fin de la mercadotecnia es diseñar soluciones que ayuden al hombre a alcanzar la verdad y el bien, su fin será moralmente legítimo. El medio que utiliza la mercadotecnia para conocer cómo generar esos satisfactores es la investigación de mercados, y una de sus herramientas es justo el uso de técnicas neurocientíficas. Entendidas de esta manera, las neurociencias aplicadas al mercadeo constituyen solo un medio para optimizar el proceso de toma de decisiones comerciales.

La complejidad de la práctica de las neurociencias como parte de la investigación de mercados que, como se ha planteado en esta tesis, es consecuencia del proceso comercial, radica precisamente en el riesgo de caer en un utilitarismo en el que la valoración moral de los métodos neurocientíficos se defina en función de su utilidad. De la misma manera, se puede incurrir fácilmente en un pragmatismo por el cual se argumente que "el fin justifica a los medios" y, por tanto, lo verdadero y lo correcto dependen del fin. Bajo este argumento, si la información que obtengo mediante métodos neurocientíficos deriva en la configuración de un producto que genere adicción, sería válido siempre y cuando cumpla con el objetivo que busco (que es incrementar ventas o alcanzar una posición favorable en el mercado en relación con mi competencia). 
En una tercera instancia, sería necesario analizar las circunstancias bajo las cuales se ponen en práctica las neurociencias. Es precisamente en este último punto en el que entran en juego los diferentes códigos de ética que existen en la industria. A fin de delimitar nuestra área de estudio, incluiremos en esta crítica únicamente el marco regulatorio del Código de Ética de la Neuromarketing Science and Business Association (NMSBA), el cual es aplicable al ámbito de los negocios y pretende establecer estándares éticos para la industria de la investigación en neuromarketing.

\section{Código de Ética de la Neuromarketing Science and Business$$
\text { Association (nmsba, 2016) }
$$

La Neuromarketing Science and Business Association agrupa a expertos en mercadotecnia e investigación de mercados alrededor del mundo, quienes tienen en común el interés en la práctica, la promoción y el conocimiento de técnicas de neuromarketing. La adopción del código de ética de la NMSBA es condicionante para ser miembro de esta asociación y parte de tres principios fundamentales, contenidos en el artículo primero:

1.1 Cumplir con los más altos estándares de investigación vigentes y utilizar los principios científicos aceptados en los países donde se lleve a cabo la práctica de neuromarketing.

1.2 No realizar acciones que puedan perjudicar la reputación y la integridad de la profesión del investigador de neuromarketing.

1.3 Entregar a los clientes los resultados sin exagerar o tergiversar las ideas más allá de lo que está científicamente aceptado. (NMSBA, 2016)

En el primer principio, en el cual se exige alinearse con los estándares de investigación y los principios científicos aceptados en cada país, encontramos demasiada apertura, al punto que puede incluso caerse en el relativismo. Es decir, si bien en algunos países los modelos de los investigadores pueden cumplir con un rigor científico y ético adecuado, es posible que en otros no sea así. La ética, al partir de principios universales, no tendría que estar condicionada a elementos circunstanciales como puede ser la ubicación geográfica o el desarrollo de una industria. Legalmente sí resulta sencillo adoptar las prácticas propias de cada ubicación en la que se desarrolle la investigación, puesto que apegarse a la ley implicará apegarse a la justicia social; sin embargo, desde una perspectiva ética, no es suficiente y no necesariamente es moralmente justo. Lo que tendría que procurarse es mantener un equilibrio entre lo que se puede hacer y lo que se debe hacer, sin dejar de lado que la prioridad es respetar en todo momento los derechos del hombre independientemente de la sociedad o la cultura en que se desarrolle (RamosZúñiga, 2014).

Los siguientes dos principios nos hablan de la defensa de la verdad, es decir, de llevar a cabo las acciones del investigador de neuromarketing con atención al cuidado de la integridad del cliente, de los otros profesionales en la industria y de sí mismo. Algo que salta a la vista al ver estos tres principios es que en ninguno de ellos se menciona la importancia de salvaguardar la seguridad y la libertad del sujeto de estudio del neuromarketing, y de recordar cómo para que la libertad esté presente se requieren dos cosas: la indeterminación de los actos de la voluntad y el dominio actual de dichos actos (López, 2006). Una persona determinada - consciente o inconscientemente- a actuar de determinada forma por circunstancias implícitas o explícitas, no podría nunca ser participante de un estudio de mercado. Si bien más adelante se habla del tema en algunas otras cláusulas, me parece que es justo este el que debería dar pie a los demás artículos contenidos en el Código de Ética.

Con respecto a la integridad de los participantes, se establecen tres normativas en el artículo segundo:

2.1 Es necesario tomar todas las precauciones razonables para asegurar que los participantes no resulten perjudicados o estresados como resultado de su participación en un proyecto de investigación de neuromarketing.

2.2 Está prohibido engañar a los participantes o aprovecharse de su falta de conocimiento de la neurociencia.

2.3 No podrá realizarse ninguna oferta de venta como resultado directo de la participación de un participante en un proyecto.

2.4 Los investigadores de neuromarketing deberán ser honestos acerca de sus habilidades y experiencia.

En el artículo 2.1 se hace referencia a la necesidad de tomar "precauciones razonables" para 
evitar que el participante sea sujeto de estrés o perjuicio, no obstante, no se habla de cuáles pueden ser dichas precauciones, pues existen algunos métodos que podrían catalogarse como invasivos, como, por ejemplo, la resonancia magnética funcional, la cual facilita la detección e identificación de áreas del cerebro durante su actividad y permite la evaluación de regiones responsables de la sensorialidad, la motricidad, la cognición y los procesos afectivos (David, Blamire \& Breiter, 1994). A fin de llevar a cabo la fmRI se solicita que el participante permanezca inmóvil dentro de una máquina tubular que puede generar ansiedad claustrofóbica.

Las fracciones 2.2, 2.3 y 2.4 enfatizan en la relevancia de actuar con honestidad en relación con los procedimientos, la finalidad de los estudios y la capacidad de los investigadores. Es frecuente encontrar a falsos "gurús" que se dicen expertos en neuromarketing, cuando realmente lo que hacen es utilizar argumentos de venta artificiosos que no se encuentran respaldados por un conocimiento y una experiencia real en el uso de las técnicas de neurociencias. Al ser el neuromarketing una integración de distintas disciplinas, es imposible pensar que existe una persona completamente capaz de esclarecer el comportamiento del hombre al construir por sí solo un modelo complejo que contemple diversas variables, lo cual verdaderamente es lo que exige la utilización de las neurociencias (Tetaz, 2014). Changeux coincide con esta tesis y afirma que las neurociencias deben permanecer en un constante contacto interdisciplinar, por lo cual su alcance debe aplicarse a todas las cuestiones humanas con una visión humanista, ética y social (Changeux, 2005). Con el fin de responder a esta necesidad, en las últimas dos décadas han surgido ciencias interdisciplinarias como, por ejemplo, la psicofisiología, cuyo objetivo es correlacionar los procesos cognitivos con las reacciones corporales (Donchin, 1981). Las empresas de neuromarketing que han sido acreedoras de prestigio en el medio son justo aquellas que ofrecen un equipo interdisciplinar: antropólogos, psicólogos, neurólogos, biólogos, actuarios, mercadólogos, etc.

El artículo tercero describe aspectos relacionados con la credibilidad:

3.1 Las preocupaciones o críticas acerca de los proyectos de neuromarketing conocidos públicamente se deberán presentar y poner a consideración de la NMSBA antes que exponerse por cualquier otro medio.

3.2 Quienes utilicen técnicas que recogen imágenes cerebrales funcionales deberán estructurar y revelar un protocolo cuya finalidad sea enfrentar hallazgos incidentales.

Es adecuado que las críticas que puedan surgir en relación con otros trabajos se den a conocer, en primer lugar, a un órgano regulador como la NMSBA, el cual fungirá como auditor para indagar en la validez de los procesos científicos o de los hallazgos resultantes de algún estudio. De esta forma, se asegura que los investigadores que puedan incurrir en alguna acción equívoca pero la cual no conlleve consigo el dolo, tengan la oportunidad de rectificar antes de que su prestigio se vea comprometido. En lo concerniente al artículo 3.2 , se exige la presencia de un protocolo cuando la investigación pretende la recolección de imágenes cerebrales funcionales, pero no se especifica cuáles son los lineamientos que deberá contener ese protocolo; tampoco se pide expresamente que una vez establecido se someta a la autorización de algún consejo médico o ético que lo avale, por lo que, desde este punto de vista, cualquier documento podría funcionar. Lo delicado del uso de este tipo de técnicas de neuroimagen es que permiten acceder con facilidad a aspectos inconscientes; por ejemplo, se puede apreciar que en ciertas situaciones morales se presenta una gran actividad en zonas que desempeñan un papel crucial en el procesamiento de las emociones. Este circuito va desde el lóbulo frontal hasta el sistema límbico (Cortina, 2010), razón por la cual cuando estamos expuestos a una disyuntiva moral las emociones juegan un papel decisivo en la decisión final que se tome.

El artículo cuarto se centra en la transparencia, y señala:

4.1 La participación en un proyecto de investigación de neuromarketing siempre será totalmente voluntaria.

4.2 Los investigadores deberán contar con una página web pública donde se describa su portafolio de servicios, así como la experiencia y credenciales académicas de sus miembros. De igual forma, se espera que publiquen una dirección física donde los responsables de la empresa puedan ser contactados. 
4.3 Los clientes cuentan con autorización expresa para supervisar el proceso por el que ideas se levantan y procesan.

4.4 Los proyectos deben ser creados, entregados, documentados e informados con transparencia para que los clientes sean capaces de comprender su alcance y trascendencia.

Como se puede observar, en términos de transparencia el Código de Ética resulta claro. Se menciona que los sujetos de estudio deben participar de manera voluntaria y, aunque en muchos de los casos la participación de los reclutados se condiciona a algún incentivo, ellos deberán colaborar sin que exista un carácter coercitivo. Esto es importante para que realmente exista un acto humano, pues cuando los actos y los pensamientos son voluntarios se atribuyen directamente a la persona, no al cerebro ni a las facultades intelectivas o sensitivas (Sanguineti, 2014). De igual manera, este mismo artículo se asegura de que el investigador principal asuma la responsabilidad del estudio al facilitar su asimilación al cliente y que, para tal efecto, lo puedan contactar en cualquier momento. Nos habla de verdad, pero también de consecuencias.

El artículo quinto explica la importancia del consentimiento:

5.1 Se solicita que las herramientas que estarán involucradas en cada estudio se expliquen a los participantes en términos sencillos, con un lenguaje simple, alejado de tecnicismos.

5.2 Antes de dar su consentimiento, los participantes deberán enunciar abiertamente que han comprendido los procedimientos, así como los objetivos generales de la investigación.

5.3 Los sujetos de estudio deben ser informados plenamente sobre el proyecto de manera previa a la aplicación de cualquier técnica de neuromarketing.

5.4 Incluso cuando ya inició el estudio de neuromarketing, los participantes tendrán libertad para retirarse en cualquier momento.

De nuevo, se indaga sobre el consentimiento y la comprensión de los objetivos y procedimientos; quizá se hace tanto énfasis en este aspecto porque al participar en un estudio de neuromarketing, al igual que en cualquier acto, la conciencia se cuestionará sobre si se obra bien o mal, y esto implica la aprobación o el rechazo de una acción que exige necesariamente el conocimiento previo (Ocampo,
2016). Sin embargo, a fin de que tal cuestionamiento se encuentre presente, es imperativo contar con información suficiente del contexto sobre el que se va a decidir, la cual le otorgará al participante un señorío real sobre la situación. Lo que quizá valdría la pena añadir a este artículo sería la necesidad de leer y entregar al participante un documento en el que se especifiquen los objetivos de investigación, los procedimientos a los que serán expuestos, el tiempo de duración de estos y el uso que se le dará a sus datos (tanto a los personales como a la información derivada de las metodologías aplicadas). Lo que actualmente sucede es que en muchas ocasiones toda esta explicación solo se difunde de manera verbal, pero al ser distintos investigadores los que están involucrados en un proyecto, cada uno de ellos maneja la información de manera distinta, por lo que los participantes no alcanzan una comprensión homogénea o total de las implicaciones de su colaboración.

La privacidad se asegura en el artículo sexto:

6.1 Los investigadores deben corroborar que los participantes sean completamente conscientes de la finalidad del proyecto.

6.2 Cada empresa y/o investigador debe redactar una política de privacidad, la cual tiene que encontrarse disponible públicamente de forma que resulte accesible a todos los participantes del estudio.

6.3 La identidad de los participantes se mantendrá oculta para el cliente, a menos que dichos participantes firmen un consentimiento donde se manifieste lo contrario.

6.4 La información personal únicamente será utilizada para los fines de investigación propios del estudio.

6.5 La información obtenida no será almacenada más tiempo del estrictamente necesario para cumplir con los objetivos del proyecto.

6.6 Los investigadores habrán de utilizar las medidas de seguridad necesarias para resguardar el acceso a la información.

6.7 Los datos de la investigación obtenidos, incluyendo escáneres cerebrales y datos del cerebro seguirán siendo propiedad de la empresa de investigación y no será compartida.

Si bien este artículo cuenta con todas las descripciones propias para garantizar que se respete la privacidad de los sujetos de estudio en cuanto 
al manejo de su identidad y datos obtenidos de la investigación, pueden detectarse dos alternativas de mejora en las fracciones quinta y séptima. En la fracción quinta se sugiere almacenar la información el tiempo necesario para cumplir los objetivos del proyecto, lo cual se deja a completo juicio del investigador. De igual forma, en el séptimo inciso se hace una excepción de la privacidad de la data, debido a que se le conceden a la empresa investigadora los derechos sobre la información obtenida como resultado de la aplicación de estudios de escáner cerebral (en este supuesto caerían los datos de la frecuencia magnética funcional y de electroencefalografías). Lo que no queda del todo explícito es la justificación para hacer tal distinción, lo cual es grave desde el punto de vista de Sanguineti, quien explica cómo un componente que se debería definir en la práctica de la neuroética es el alcance del uso de técnicas de observación de las activaciones cerebrales, las cuales arrojan un conocimiento profundo de la conducta o de las inclinaciones de las personas, aun cuando su fin apunte a la mejora de las prestaciones humanas (Sanguineti, 2015). Afirmar únicamente que los datos obtenidos de neuroimágenes no se compartirán, pero que son propiedad del investigador, no es suficiente para definir su alcance, puesto que esta postura abre la puerta a utilizarlos con cualquier fin, siempre y cuando las aplicaciones se lleven a cabo dentro de la organización que los generó. Incluso, Focquaert mantiene la tesis según la cual de manera posterior a cualquier intervención médica - como puede ser un escaneo cerebrales posible alterar la individualidad de las personas, puesto que no es posible aislar el cerebro de una persona para su estudio, además de que nuestras experiencias y formas de pensar dependen en gran medida de lo que suceda en nuestro cuerpo (Focquaert, 2003).

En el artículo séptimo se detallan los derechos de los participantes:

7.1 Los sujetos de estudio deberán confirmar de manera explícita que son conscientes de que no están obligados a participar en el proyecto.

7.2 Los participantes podrán retirarse de la investigación en cualquier momento.

7.3 Se habrá de garantizar a los participantes que sus datos personales no se entregarán a terceros.

7.4 Se garantizará a los sujetos de estudio que sus ideas pueden ser eliminadas o modificadas conforme a su petición.
7.5 Es necesario cuidar que se mantengan las normas de protección de datos personales de los participantes aun cuando exista una transferencia de dichos datos entre países, por ejemplo, cuando los datos se recolecten en un país y se analicen en otro. Cuando esto sucede, los principios de protección de datos contenidos en el código de la NMSBA deben ser respetados.

Aunque el artículo séptimo pudiera resultar repetitivo en relación con los artículos cuarto, quinto y sexto, se agrega al Código un aspecto nuevo, el cual consiste en asegurar que se cuide la privacidad de los datos de los participantes aunque se trabaje mediante un sistema de colaboración en redes, lo cual es habitual hoy en día.

Los niños y los jóvenes constituyen grupos de estudio vulnerables, razón por la cual se hace especial referencia a ellos en el artículo octavo, en el cual se establece que los estudios de neuromarketing con participantes menores de 18 años solo se llevarán a cabo una vez que se cuente con el consentimiento informado de los padres de los participantes. La protección de grupos vulnerables ha sido objeto de especial atención por parte de la Asociación Médica Mundial, e incluso implicó una revisión continua de la Declaración de Helsinski, la cual establece pautas éticas para los médicos involucrados en investigación biomédica. Esta declaración se ha perfeccionado con respecto a la inclusión en investigación biomédica de sujetos que presenten alguna vulnerabilidad, pues se sabe que, en caso de ser necesario su estudio, deben minimizarse los riesgos de participación y maximizarse los beneficios sociales de los resultados (Gaudlitz, 2008). A este artículo agregaría la obligación de entregar a los padres o tutores una responsiva firmada por parte del responsable del estudio acompañada de una grabación en audio o video que funja como evidencia del procedimiento al que se vio sujeto el participante, lo cual contribuiría a proteger con un empeño aun mayor su integridad.

Dado que es usual en la industria de la investigación de mercados la necesidad de trabajar con proveedores externos, el artículo noveno hace énfasis en que los investigadores de neuromarketing deben comunicarle al cliente antes de comenzar el trabajo si alguna parte del proyecto será subcontratada fuera de la empresa, lo que incluye la colaboración de asesores o consultores externos. Con esto se contribuye a cumplir el principio de justicia, otorgándoles a todos los clientes un 
acceso equitativo a los riesgos y beneficios en los que pueden incurrir una vez que decidan confiar tanto en la empresa que contratan como en todos los proveedores involucrados en el transcurso de este proceso.

Parte natural del proceso de investigación científica deriva en la publicación de los hallazgos de los estudios, por tanto, el artículo décimo indica que, cuando los resultados de un proyecto se comparten públicamente, los investigadores habrán de definir con claridad qué parte del informe muestra los resultados clave. De igual manera, se propone que los investigadores no asocien su nombre a un proyecto en particular, a menos que hayan participado activamente en él y se encuentren facultados para defender los hallazgos. Resulta interesante que se aclare en este artículo la imposibilidad de utilizar en la publicación el nombre de autores que no hayan participado en la elaboración de la investigación, lo cual desafortunadamente es una incidencia no ética que constituye un plagio y se presenta con cierta frecuencia en el afán de alcanzar los índices de impacto solicitados por organismos de acreditación de la actividad investigadora. En este sentido, habría que complementar estos errores de investigación con el conocimiento de cómo si bien es grave el plagio, también es posible cometer fraude científico cuando se "fabrica" una parte o la totalidad del estudio y se manipulan los datos con el fin de favorecer la hipótesis de estudio (Laucirica-Hernández, 2007).

El artículo onceavo pone de manifiesto el compromiso implícito entre los miembros de la NMSBA de aplicar constantemente el Código de Ética y asegurarse de que sus propios clientes, proveedores, asesores y participantes de los estudios cumplan con los requisitos propios de su rol. Se especifica que faltar al cumplimiento de este Código implicará la terminación de su membresía en la NMSBA, por lo cual, de esta forma, se blinda a la comunidad que pertenece a esta asociación.

Finalmente, el artículo doceavo esgrime argumentos relativos a la aplicación del Código al indicar que tanto los investigadores como sus clientes deben reconocer el Código de Ética de la NMSBA y, de manera adicional, deberán apegarse a otras normas de autorregulación que pudieran resultar relevantes para una región o proyecto en particular. También subraya que se da por hecho que el Código de Ética lo conocen todos los asociados, ya que se encuentra disponible al público en la página web de la NMSBA. Me parece oportuno el cierre de este Código, el cual apuntala su aplicabilidad a todos los miembros de la NMSBA y sus alcances normativos, e indica la trascendencia de su complementariedad con el contexto particular de la práctica de investigación de mercados o neurociencias en cada país. Al tratar específicamente de México, sería necesario revisar los estatutos de actuación ética establecidos por la Asociación Mexicana de Agencias de Investigación de Mercados y Opinión Pública (AMAI).

\section{Conclusiones}

Después de realizar una revisión concienzuda de los diferentes elementos que conforman el Código de Ética de la Neuromarketing Science and Business Association, es claro que la aplicación de este Código es oportuna en un ambiente de negocios; sin embargo, puede no resultar suficiente en el propósito de regular la inclusión de seres humanos en investigación psicofisiológica.

Estudiar a personas es propio de la búsqueda del progreso de la ciencia, no obstante, un verdadero progreso en un sentido humanístico no solo implica el entendimiento de las condiciones físicas y biológicas del hombre, sino que también habrá de concebirse como un medio de forjar la personalidad y el carácter (Llano \& Zagal, 2001). El dilema radica entre la valoración del beneficio del conocimiento en relación con el medio de obtención de este que, en las neurociencias, son los seres humanos.

Resulta imperioso recordar que lo posible tecnológicamente no necesariamente es válido éticamente, por lo que en cualquier código de ética que implique la investigación con personas tendría que garantizarse la preservación de su integridad. El Informe de Belmont, redactado por la Comisión Nacional para la Protección de los Sujetos Humanos de Investigación Biomédica y Comportamental, detalla tres principios claves que deben cuidarse en la ética de investigación con humanos y, a nuestro juicio, deberían adoptarse en cualquier código de investigación neurocientífica: el principio de respeto por las personas, por el cual se cuida el derecho del participante a decidir libremente su participación en un estudio e implica un consentimiento informado; el principio de beneficencia, el cual custodia el bienestar del sujeto de investigación al minimizar los riesgos del procedimiento; $y$, finalmente, el principio de justicia, por el cual se busca que los participantes en el estudio obtengan beneficios de 
este y que su selección sea equitativa (Comision Nacional para la Protección de Sujetos Humanos de Investigación Biomédica y Comportamental, 2003). Al considerar estos tres principios, considero que en el Código de Ética de la NMSBA habría que defender la observancia de los procedimientos a los que son sujetos los participantes y, de igual forma, asegurar que obtengan un beneficio real por los resultados del estudio, el cual no necesariamente tendría que darse como individuo, pero sí como miembro de una sociedad. Utilizar los resultados de los estudios únicamente con objetivos monetarios de la compañía contratante sería privilegiar el fin a costa del medio, lo cual resulta aberrante si se considera que justo ese medio es un ser humano.

En futuras investigaciones valdría la pena desentrañar otros códigos de ética existentes en la industria de la investigación neurocientífica con fines comerciales, a fin de identificar cuáles puntos comparten entre sí y de cuáles principios básicos adolecen, y se lleven así a un comparativo con códigos de ética propios de la investigación biomédica.

\section{REFERENCIAS}

Beckman, S. (2008). Interpretive consumer research. Dinamarca: Copenhagen Business School.

Changeux, J.-P. (2005). El hombre de verdad. México: Fondo de Cultura Económica.

Comins-Mingol, I. \& París-Albert, S. (2013). Un análisis desde la filosofía para la paz. Convergencia, Revista de Ciencias Sociales, 62, 107-133.

Comisión Nacional para la Protección de Sujetos Humanos de Investigación Biomédica y Comportamental. (2003). Informe Belmont: Principios y guías éticos para la protección de los sujetos humanos de investigación. National Institutes of Health, 12.

Cortina, A. (2010). Neuroética: ¿Las bases cerebrales de una ética universal con relevancia política? Isegoría, (42), 129-148.

David, A., Blamire, A. \& Breiter, H. (1994). Functional magnetic resonance imaging: a new technique with implications for psychology and psychiatry. Br J Psychiatry, 164(2), 7.

Denton, D. (2009). El despertar de la consciencia. España: Paidós.
Donchin, E. (1981). Surprise!... Surprise? The Society for Psychophysiological Research, 18(5), 493-513.

Filser, M. (2000). Confiance et comportement du consommateur. Dijon, Francia: Université de Bourgogne.

Focquaert, F. (2003, enero). Personal identity and its boundaries: philosophical thought experiments. Philosophica, 72, 131-152.

Gaudlitz, M. (2008). Reflexiones sobre los principios éticos en investigación biomédica en seres humanos. Revista Chilena de Enfermedades Respiratorias, $24(2), 138-142$.

Laucirica-Hernández, C. (2007). Ética de la publicación científica. Revista Habanera de Ciencias Médicas, $6,1-7$.

Llano, C. \& Zagal, H. (2001). El rescate ético de la empresa y el mercado. México: Trillas.

López, J. G. (2006). Escritos de antropología filosófica ( $2^{\underline{a}}$ ed.). España: Eunsa Ediciones Universidad de Navarra S. A.

Neuromarketing Science and Business Association-NMSBA. (2016). Código de ética de la NMSBA. Recuperado de http://www.nmsba.com/ethics/ spanish

Ocampo, M. (2016). La absurda negación del orden moral propuesta por el relativismo. Recuperado de http://infocatolica.com/blog/concordia.php/ 1609280646-la-absurda-negacion-del-orden

Plassmann, H., Ramsøy, T. Z. \& Milosavljevic, M. (2012). Branding the brain: A critical review and outlook. Journal of Consumer Psychology, 22(1), 18-36. doi: http://doi.org/10.1016/j.jcps.2011.11.010

Ramos-Zúñiga, R. (2014). La neuroética como una nueva perspectiva epistemológica en neurociencias. Revista de Neurología, 58(4), 145-146.

Rosenzweig, M. R. \& Leiman, A. I. (2005). Psicología fisiológica. Bogotá: Mc Graw Hill.

Roskies, A. (2002). Neuroethics for the new millenium. Neuron, 35(1), 21-23.

Santo Tomás. (s. f.). Suma Teológica (p. p.I, q.84, a4.). Madrid: Edición dirigida por los Regentes de Estudios de las Provincias Dominicanas en España.

Sanguineti, J. J. (2014). Neurociencia y filosofía del hombre. España: Palabra.

Sanguineti, J. J. (2015). El desafío antropológico de las neurociencias. Neurociencia, filosofía y teología. Rivista Di Scienze dell'Educazione, 53(3), 383-400.

Tetaz, M. (2014). Psychonomics: la economía está en tu mente. Argentina: Ediciones B.

Zaltman, G. (2003). How customers think: essential insights into the mind of the market. Boston, Massachusetts: Harvard Business School Press. 